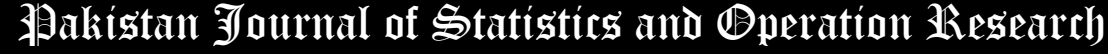

\section{Goodness of Fit Tests for Marshal-Olkin Extended Rayleigh distribution}

* Corresponding Author

\author{
Naz Saud ${ }^{1 *}$, Sohail Chand ${ }^{2}$
}

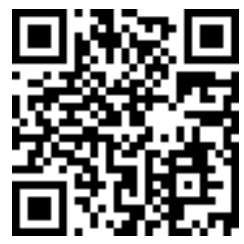

1. Lahore College for Women University, Lahore, Pakistan, naz.saud17@gmail.com

2. College of Statistical and Actuarial Sciences, University of the Punjab, Lahore Pakistan, sohail.stat@gmail.com

\begin{abstract}
A class of goodness of fit tests for Marshal-Olkin Extended Rayleigh distribution with estimated parameters is proposed. The tests are based on the empirical distribution function. For determination of asymptotic percentage points, Kolomogorov-Sminrov, Cramer-von-Mises, Anderson-Darling, Watson, and Liao-Shimokawa test statistics are used. This article uses Monte Carlo simulations to obtain asymptotic percentage points for Marshal-Olkin extended Rayleigh distribution. Moreover, power of the goodness of fit test statistics is investigated for this lifetime model against several alternatives.
\end{abstract}

Key Words: Marshal-Olkin Extended Rayleigh distribution, Goodness of fit, Monte-Carlo simulation, Asymptotic percentage points, Power test.

\section{Introduction}

The Rayleigh distribution is very popular among lifetime distributions. Some of the areas where it is used are the study of vibrations and waves, theory of communication to explain instantaneous peak power and hourly median of signals received at a radio, to model wind speed under certain circumstances at wind turbine sites in a year and for modeling the lifetimes of devices. There are variety of methods to add parameters to some existing probability distribution to get some new distribution, which generally provides much more flexibility to model the lifetime data. Marshall and Olkin (1997) suggested that a new lifetime distribution having survival function $\bar{F}(x ; \alpha)$ can be formed by adding a parameter " $\alpha$ " to another distribution $G(x)$. i.e.

$$
\bar{F}(x, \alpha)=\frac{\alpha \bar{G}(x)}{1-\alpha G(x)} \quad, \quad x \in R, \alpha>0
$$

where $\bar{\alpha}=1-\alpha$, and $\bar{G}(x)$ is the survival function of a continuous type. They discussed this method with the application to the Exponential and Weibull families.

Goodness of fit tests evaluate the degree of agreement between the observed sample distribution and the theoretical distribution. There are so many goodness of fit tests available in the literature, some of which endure severe limitations. Grouping of data is requirement of chi-square goodness of fit test which do not work in case of smaller sample. Tests based on correlations and tests based on moment ratios are most of the times under-estimated.

In most of practical situations, the parameters are usually unknown and are estimated from sample data, using an estimation method such as maximum likelihood. Goodness of-fit test statistics are not distribution-free when the parameters are unknown and have to be estimated from sample. The distribution of test statistics depend on sample size, the population parameters being estimated, estimation technique and on the hypothesized distribution.

Empirical distribution function (EDF) based tests are appropriate to use when the population parameters are unknown and are estimated from sample data, as these provide high power than other tests. Moreover, the empirical 
distribution functions based goodness of fit tests give equal weight to discrepancy between theoretical distribution and empirical distribution functions consequent to all observations.

Many researchers such as Lilliefors (1967), Lilliefors (1969), Woodruff et al. (1984), and Yen and Moore (1988) have used different test statistics to the case where parameters are unknown and to be estimated from sample. Recently, the goodness of fit for several distributions have been studied by many authors. Such as, for Generalized Pareto distribution Choulakian and Stephens (2001) used Cramer-von Mises and Anderson Darling test statistics to test goodness-of-fit and power of GP distribution against other distributions. Hassan (2005) investigated goodness of fit of Generalized Exponential distribution which was originally introduced by Gupta and Kundu (1999). Goodness of Generalized Frechet distribution was studied by Abd-Elfattah et al. (2010). They used Kolmogorov-Smirnov, Cramervon Mises, Anderson Darling, Watson and Liao and Shimokawa statistics to test goodness of fit. Abd-Elfattah (2011) tested Generalized Rayleigh distribution for goodness of fit when the parameters were not known. Shin et al. (2011) evaluated the goodness of Generalized Extreme Value distribution and Generalized Logistic distribution with extension to shape parameter. For Gumbel distribution, goodness of fit was investigated by Zainal Abidin and Midi (2012) using six goodness of fit statistics. Al-Zahrani (2012) used Kolmogorov-Smirnov, Cramer-von Mises, Anderson-Darling, Watson and Liao and Shimokawa statistic to test the goodness of fit of Topp-Leone distribution when parameter were not known.

This article is focused on the goodness of fit testing of Marshall-Olkin Extended Rayleigh distribution $\operatorname{MOR}(\alpha, \beta)$ which is obtained from Cordeiro and Lemonte (2013) for $\lambda=\beta^{2}$ and $\gamma=2$. MOR distribution is right skew, symmetrical and left skew depending upon values of parameters. This new distribution has wide applications due to its characteristic of modeling the lifetime data with increasing and modified upside-downbathtub failure rate. We have assessed the performance of this new lifetime model using five important EDF tests. These are Kolmogorov Sminrov, Cramer-Von Mises, Anderson Darling, Watson and Liao and Shimokawa test statistics. We have computed asymptotic percentage points also known as critical values of these goodness of fit test statistics using Monte-Carlo simulations. We used Newton-Raphson iterative method to obtain maximum likelihood estimates of unknown parameters of Marshall-Olkin Extended Rayleigh distribution. We have also calculated power of these test statistics for the proposed distribution against six competitive probability distributions. All simulations are performed in $R$.

This article is organized as follows. In section 2, we present estimation of the parameters by maximum likelihood for $\operatorname{MOR}(\alpha, \beta)$ distribution. Simulations and goodness of fit tests are discussed in section 3 and 4 . Section 5 is devoted to the calculations of critical values for the test statistics, while power comparison is given in section 6. Real data application is presented in section 7. Finally, we conclude the paper in section 8.

\section{Estimating the parameters of Marshal-Olkin extended Rayleigh distribution}

A two parameter distribution, named as Marshal-Olkin Extended Rayleigh (MOR) distribution is obtained by using equation (1) as a generalization of standard Rayleigh distribution. Let ' $\mathrm{X}$ ' be a continuous random variable follows the Marshal-Olkin Extended Rayleigh (MOR) distribution with shape parameter a and scale parameter $\beta$, the cumulative distribution function for MOR distribution is

$$
F(x)=\frac{1-e^{-(\beta x)^{2}}}{1-\bar{\alpha} e^{-(\beta x)^{2}}}, \quad x>0, \quad \alpha, \beta>0 \text { where } \bar{\alpha}=1-\alpha .
$$

The density function of Marshal-Olkin Extended Rayleigh (MOR) distribution is

$$
f(x)=\frac{2 \alpha \beta^{2} x e^{-(\beta x)^{2}}}{\left(1-\bar{\alpha} e^{-(\beta x)^{2}}\right)^{2}}, \quad x>0, \alpha, \beta>0 .
$$




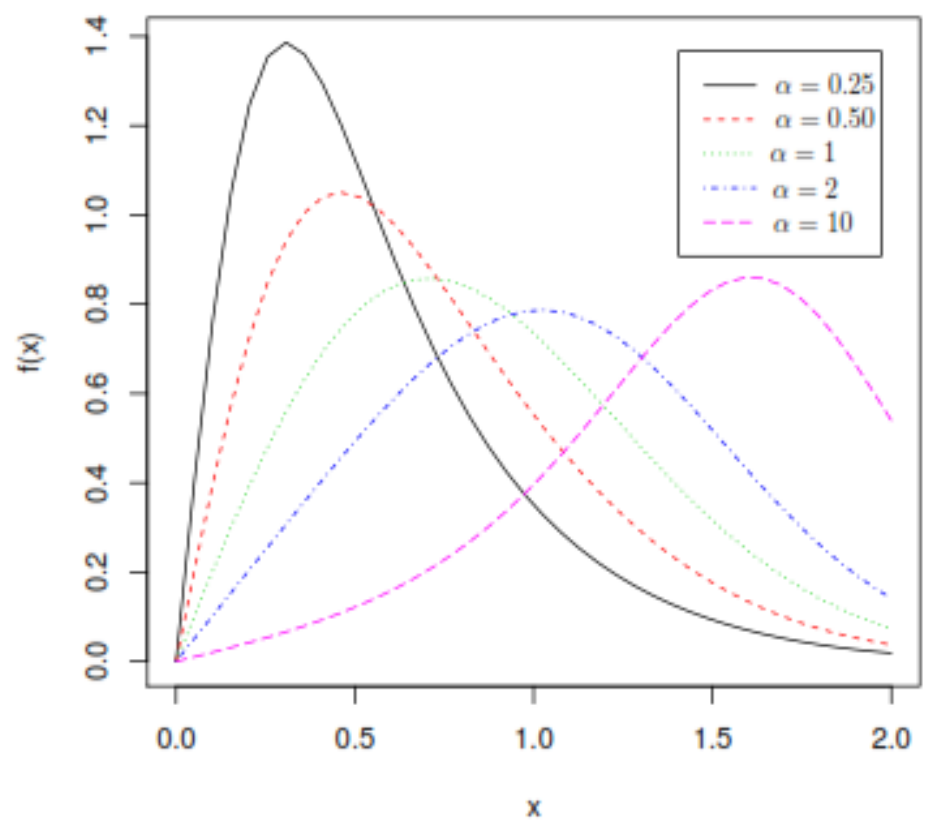

Figure 1. Probability density function of Marshal-Olkin Extended Rayleigh distribution for $\beta=1$ and different values of shape parameter $\alpha$.

Let $X_{1}, X_{2}, \ldots, X_{n}$ be a random sample from Marshal-Olkin Extended Rayleigh (MOR) distribution, then the loglikelihood function is

$$
L=n \ln 2+n \ln \alpha+2 n \ln \beta+\sum_{i=1}^{n} \quad \ln x_{i}-\sum_{i=1}^{n} \quad\left(\beta x_{i}\right)^{2}-2 \sum_{i=1}^{n} \quad \ln \left(1-\bar{\alpha} e^{-\left(\beta x_{i}\right)^{2}}\right)
$$

The normal equations are

$$
\begin{aligned}
& \frac{\partial \ln L}{\partial \alpha}=\frac{n}{\alpha}-2 \sum_{i=1}^{n}\left[\frac{e^{-(\beta x)^{2}}}{1-(1-\alpha) e^{-(\beta x)^{2}}}\right]=0 \\
& \frac{\partial \ln L}{\partial \beta}=\frac{2 n}{\beta}-2 \beta \sum_{i=1}^{n} x_{i}^{2}-4 \sum_{i=1}^{n}\left[\frac{(1-\alpha) \beta x_{i}^{2} e^{-(\beta x)^{2}}}{1-(1-\alpha) e^{-(\beta x)^{2}}}\right]=0
\end{aligned}
$$

The MLE's of $\alpha$ and $\beta$ can be determined from the solution of non-linear equations (5) and (6). Since the MLE's are not in explicit form so we use Newton-Raphson iterative method to obtain the numerical estimates of the parameters of MOR distribution.

2.1. Random Number Generator. The random number generator of Marshal-Olkin Extended Rayleigh (MOR) distribution is given by

$$
x=\frac{1}{\beta} \sqrt{\ln \left(\frac{R \bar{\alpha}-1}{R-1}\right)}
$$

where $\mathrm{R}$ is the random number from uniform distribution $\mathrm{u}(0,1)$.

\section{Simulations and Power Study for Marshal-Olkin extended Rayleigh distribution}

We have assessed the performance of the proposed lifetime model using five important goodness of fit tests. For this purpose, we have computed critical values of these goodness of fit test statistics using Monte-Carlo simulations. We used Newton-Raphson iterative method to obtain maximum likelihood estimates of unknown parameters of MarshalOlkin Extended Rayleigh distribution. We have also calculated power of these test statistics for MOR distribution against six competitive probability distributions.

\section{Goodness of Fit Tests}


Goodness of fit tests are used to assess whether a particular data set is consistent with a hypothesized null distribution or not. We use five important EDF tests. These are Kolmogorov Sminrov, Cramer-Von Mises, Anderson Darling, Watson and Liao and Shimokawa test statistics. To test the null hypothesis,

$H_{0}$ : The random sample $X_{1}, X_{2}, \ldots, X_{n}$ come from Marshal-Olkin Extended Rayleigh distribution (3) with unknown parameters, we used the following formulas of the test statistics for computational purpose :

1. The Kolomogorov Smirnov (KS) test statistic $D_{n}$ is

$$
D_{n}=\max \left[\frac{i}{n}-F_{0}\left(x_{i}, \hat{\alpha}, \hat{\beta}\right), F_{0}\left(x_{i}, \hat{\alpha}, \hat{\beta}\right)-\frac{i-1}{n}\right]
$$

Where $F_{0}\left(x_{i}, \hat{\alpha}, \hat{\beta}\right)$ is the cumulative distribution function of $\operatorname{MOR}(\alpha, \beta)$ distribution, $n$ is the sample size and $\hat{\alpha}, \hat{\beta}$ are the estimated parameters using maximum likelihood estimators of $\alpha$ and $\beta$ from (5) and (6), respectively.

2. The Cramer-von-Mises (CVM) test statistic $W_{n}^{2}$ is

$$
W_{n}^{2}=\frac{1}{12 n}+\sum_{i=1}^{n}\left[F_{0}\left(x_{i}, \hat{\alpha}, \widehat{\beta}\right)-\frac{2 i-1}{2 n}\right]^{2}
$$

3. The Anderson-Darling (AD) test statistic $A_{n}^{2}$ is

$$
A_{n}^{2}=-n-\frac{1}{n} \sum_{i=1}^{n}(2 i-1)\left[\ln F_{0}\left(x_{i}, \hat{\alpha}, \hat{\beta}\right)+\ln \left(1-F_{0}\left(x_{n+1-i}, \hat{\alpha}, \hat{\beta}\right)\right)\right]
$$

4. The Watson $U_{n}^{2}$ test statistic is

$$
U_{n}^{2}=W_{n}^{2}-n\left[\frac{\sum_{i=1}^{n} F_{0}\left(x_{i}, \widehat{\alpha}, \widehat{\beta}\right)}{n}-\frac{1}{2}\right]^{2}
$$

5. The Liao and Shimokawa (LS) test statistic is

$$
L_{n}=\frac{1}{\sqrt{n}} \sum_{i=1}^{n} \frac{\max \left[\frac{i}{n}-F_{0}\left(x_{i}, \widehat{\alpha}, \widehat{\beta}\right), F_{0}\left(x_{i}, \widehat{\alpha}, \widehat{\beta}\right)-\frac{i-1}{n}\right]}{\sqrt{F_{0}\left(x_{i}, \widehat{\alpha}, \widehat{\beta}\right)\left[1-F_{0}\left(x_{i}, \widehat{\alpha}, \widehat{\beta}\right)\right]}}
$$

\section{Calculation of critical values for Marshal-Olkin extended Rayleigh distribution}

For calculating critical values, we proceeded as follows:

1. We generated a random sample $X_{1}, X_{2}, \ldots, X_{n}$ from Marshal-Olkin Extended Rayleigh distribution with probability density function (3).

2. For this, we first generated a random sample of n ordered statistics i.e. $U_{(1)}, U_{(2)}, U_{(3)}, \ldots \ldots, U_{(n)}$ from uniform distribution $u(0,1)$.

3. Then using (7), the random number generator of $\operatorname{MOR}(\alpha, \beta)$ with $\beta=1$ and $\alpha=0.50$, we obtained an ordered sample of size $\mathrm{n}$ from MOR distribution.

4. We used this random sample to estimate the unknown parameters of MOR distribution by method of maximum likelihood. Because the normal equations (5) and (6) to obtain MLE's are non-linear, so we used Newton-Raphson iterative method to solve these non-linear equations.

5. We performed this iterative procedure using the 'rootSolve' package in R that was suggested by Soetaert (2009).

6. Then we determined the cumulative distribution function of our hypothesized Marshal-Olkin Extended Rayleigh distribution using these maximum likelihood estimates of the unknown parameters.

7. We selected the sample of size as $n=5(5), 50(10), 100$ and calculate all five test statistics $D_{n}, W_{n}^{2}, A_{n}^{2}, U_{n}^{2}$ and $L_{n}$ for these sample sizes.

8. We repeated this procedure to generate 10,000 Monte-Carlo runs, from which we obtained 10,000 independent values of each test statistic.

9. We then ranked these 10,000 values for each statistic.

10. We selected seven levels of significance $(\gamma)$ as $0.01,0.025,0.05,0.10,0.15,0.20$ and 0.25 at which we compute the critical values for each test statistic against each sample size.

Following table represents the critical points of five goodness of fit test statistics against different significance level and sample sizes using Monte Carlo method.

Table 1: Critical Points of Test Statistics for Marshal-Olkin extended Rayleigh Distribution

\begin{tabular}{|c|c|c|c|c|c|c|c|c|}
\hline Sample Size & Test Statistic & \multicolumn{9}{|c|}{ Significance Level $\gamma$} \\
\cline { 3 - 9 } & & 0.01 & 0.025 & 0.05 & 0.10 & 0.15 & 0.20 & 0.25 \\
\hline \multirow{2}{*}{5} & $D_{n}$ & 0.3498 & 0.3305 & 0.3143 & 0.2935 & 0.2795 & 0.2697 & 0.2611 \\
\cline { 2 - 10 } & $W_{n}^{2}$ & 0.1386 & 0.1176 & 0.1029 & 0.0862 & 0.0766 & 0.0698 & 0.0643 \\
\hline
\end{tabular}




\begin{tabular}{|c|c|c|c|c|c|c|c|c|}
\hline & $A_{n}^{2}$ & 1.1269 & 0.8877 & 0.7380 & 0.6036 & 0.5340 & 0.4856 & 0.4480 \\
\hline & $U_{n}^{2}$ & 0.1386 & 0.1176 & 0.1029 & 0.0862 & 0.0766 & 0.0697 & 0.0643 \\
\hline & $L_{n}$ & 2.9346 & 2.1388 & 1.7308 & 1.4279 & 1.2850 & 1.2088 & 1.1561 \\
\hline \multirow[t]{5}{*}{10} & $D_{n}$ & 0.2704 & 0.2531 & 0.2388 & 0.2222 & 0.2119 & 0.2023 & 0.1946 \\
\hline & $W_{n}^{2}$ & 0.1511 & 0.1266 & 0.1078 & 0.0893 & 0.0786 & 0.0706 & 0.0649 \\
\hline & $A_{n}^{2}$ & 1.0794 & 0.8879 & 0.7494 & 0.6115 & 0.5443 & 0.4951 & 0.4534 \\
\hline & $U_{n}^{2}$ & 0.1511 & 0.1266 & 0.1078 & 0.0893 & 0.0786 & 0.0706 & 0.0649 \\
\hline & $L_{n}$ & 1.9710 & 1.5744 & 1.3632 & 1.1889 & 1.1049 & 1.0524 & 1.0104 \\
\hline \multirow[t]{5}{*}{15} & $D_{n}$ & 0.2316 & 0.2156 & 0.2024 & 0.1877 & 0.1773 & 0.1702 & 0.1641 \\
\hline & $W_{n}^{2}$ & 0.1593 & 0.1314 & 0.1123 & 0.0928 & 0.0815 & 0.0728 & 0.0668 \\
\hline & $A_{n}^{2}$ & 1.1057 & 0.9021 & 0.7732 & 0.6316 & 0.5594 & 0.5079 & 0.4665 \\
\hline & $U_{n}^{2}$ & 0.1594 & 0.1314 & 0.1123 & 0.0928 & 0.0815 & 0.0728 & 0.0668 \\
\hline & $L_{n}$ & 1.6417 & 1.4050 & 1.2447 & 1.0988 & 1.0275 & 0.9796 & 0.9412 \\
\hline \multirow[t]{5}{*}{20} & $D_{n}$ & 0.2026 & 0.1888 & 0.1770 & 0.1650 & 0.1563 & 0.1494 & 0.1443 \\
\hline & $W_{n}^{2}$ & 0.1630 & 0.1354 & 0.1140 & 0.0933 & 0.0818 & 0.0738 & 0.0675 \\
\hline & $A_{n}^{2}$ & 1.1284 & 0.9041 & 0.7697 & 0.6431 & 0.5645 & 0.5083 & 0.4688 \\
\hline & $U_{n}^{2}$ & 0.1630 & 0.1354 & 0.1139 & 0.0932 & 0.0817 & 0.0738 & 0.0674 \\
\hline & $L_{n}$ & 1.4749 & 1.2857 & 1.1532 & 1.0435 & 0.9741 & 0.9289 & 0.8943 \\
\hline \multirow[t]{5}{*}{25} & $D_{n}$ & 0.1833 & 0.1720 & 0.1614 & 0.1491 & 0.1414 & 0.1354 & 0.1303 \\
\hline & $W_{n}^{2}$ & 0.1652 & 0.1369 & 0.1144 & 0.0945 & 0.0832 & 0.0741 & 0.0676 \\
\hline & $A_{n}^{2}$ & 1.1338 & 0.9283 & 0.7876 & 0.6441 & 0.5644 & 0.5127 & 0.4729 \\
\hline & $U_{n}^{2}$ & 0.1652 & 0.1369 & 0.1144 & 0.0945 & 0.0832 & 0.0741 & 0.0676 \\
\hline & $L_{n}$ & 1.4167 & 1.2331 & 1.1173 & 1.0056 & 0.9402 & 0.8981 & 0.8666 \\
\hline \multirow[t]{5}{*}{30} & $D_{n}$ & 0.1701 & 0.1580 & 0.1482 & 0.1369 & 0.1294 & 0.1240 & 0.1195 \\
\hline & $W_{n}^{2}$ & 0.1692 & 0.1381 & 0.1175 & 0.0954 & 0.0832 & 0.0745 & 0.0680 \\
\hline & $A_{n}^{2}$ & 1.1078 & 0.9422 & 0.7906 & 0.6519 & 0.5763 & 0.5186 & 0.4774 \\
\hline & $U_{n}^{2}$ & 0.1692 & 0.1381 & 0.1175 & 0.0954 & 0.0832 & 0.0745 & 0.0680 \\
\hline & $L_{n}$ & 1.3432 & 1.1842 & 1.0791 & 0.9755 & 0.9190 & 0.8772 & 0.8456 \\
\hline \multirow[t]{5}{*}{35} & $D_{n}$ & 0.1570 & 0.1463 & 0.1374 & 0.1280 & 0.1207 & 0.1157 & 0.1112 \\
\hline & $W_{n}^{2}$ & 0.1668 & 0.1367 & 0.1164 & 0.0948 & 0.0833 & 0.0750 & 0.0683 \\
\hline & $A_{n}^{2}$ & 1.1120 & 0.9257 & 0.7890 & 0.6436 & 0.5731 & 0.5178 & 0.4777 \\
\hline & $U_{n}^{2}$ & 0.1668 & 0.1367 & 0.1164 & 0.0948 & 0.0833 & 0.0750 & 0.0683 \\
\hline & $L_{n}$ & 1.3092 & 1.1446 & 1.0411 & 0.9478 & 0.8899 & 0.8551 & 0.8240 \\
\hline \multirow[t]{5}{*}{40} & $D_{n}$ & 0.1483 & 0.1386 & 0.1286 & 0.1189 & 0.1134 & 0.1087 & 0.1044 \\
\hline & $W_{n}^{2}$ & 0.1672 & 0.1365 & 0.1155 & 0.0945 & 0.0831 & 0.0754 & 0.0685 \\
\hline & $A_{n}^{2}$ & 1.1114 & 0.9276 & 0.7849 & 0.6476 & 0.5754 & 0.5220 & 0.4794 \\
\hline & $U_{n}^{2}$ & 0.1672 & 0.1365 & 0.1155 & 0.0945 & 0.0831 & 0.0754 & 0.0685 \\
\hline & $L_{n}$ & 1.2417 & 1.1062 & 1.0241 & 0.9311 & 0.8787 & 0.8388 & 0.8087 \\
\hline \multirow[t]{5}{*}{45} & $D_{n}$ & 0.1410 & 0.1308 & 0.1223 & 0.1131 & 0.1069 & 0.1023 & 0.0985 \\
\hline & $W_{n}^{2}$ & 0.1718 & 0.1418 & 0.1173 & 0.0959 & 0.0834 & 0.0754 & 0.0686 \\
\hline & $A_{n}^{2}$ & 1.1210 & 0.9217 & 0.7816 & 0.6497 & 0.5730 & 0.5209 & 0.4786 \\
\hline & $U_{n}^{2}$ & 0.1718 & 0.1418 & 0.1173 & 0.0959 & 0.0834 & 0.0754 & 0.0686 \\
\hline & $L_{n}$ & 1.1922 & 1.0786 & 0.9949 & 0.9112 & 0.8629 & 0.8217 & 0.7932 \\
\hline \multirow[t]{5}{*}{50} & $D_{n}$ & 0.1359 & 0.1249 & 0.1165 & 0.1071 & 0.1015 & 0.0972 & 0.0935 \\
\hline & $W_{n}^{2}$ & 0.1701 & 0.1393 & 0.1172 & 0.0952 & 0.0833 & 0.0752 & 0.0681 \\
\hline & $A_{n}^{2}$ & 1.1329 & 0.9226 & 0.7805 & 0.6483 & 0.5745 & 0.5165 & 0.4768 \\
\hline & $U_{n}^{2}$ & 0.1701 & 0.1393 & 0.1172 & 0.0952 & 0.0833 & 0.0752 & 0.0681 \\
\hline & $L_{n}$ & 1.1976 & 1.0708 & 0.9813 & 0.9026 & 0.8490 & 0.8125 & 0.7836 \\
\hline \multirow[t]{4}{*}{60} & $D_{n}$ & 0.1224 & 0.1136 & 0.1059 & 0.0982 & 0.0930 & 0.0893 & 0.0862 \\
\hline & $W_{n}^{2}$ & 0.1713 & 0.1390 & 0.1165 & 0.0957 & 0.0833 & 0.0752 & 0.0685 \\
\hline & $A_{n}^{2}$ & 1.1345 & 0.9413 & 0.7858 & 0.6541 & 0.5786 & 0.5212 & 0.4807 \\
\hline & $U_{n}^{2}$ & 0.1713 & 0.1390 & 0.1165 & 0.0957 & 0.0833 & 0.0752 & 0.0685 \\
\hline
\end{tabular}




\begin{tabular}{|c|c|c|c|c|c|c|c|c|}
\hline & $L_{n}$ & 1.1658 & 1.0482 & 0.9652 & 0.8798 & 0.8316 & 0.7940 & 0.7641 \\
\hline \multirow[t]{5}{*}{70} & $D_{n}$ & 0.1141 & 0.1056 & 0.0989 & 0.0916 & 0.0869 & 0.0832 & 0.0801 \\
\hline & $W_{n}^{2}$ & 0.1657 & 0.1378 & 0.1177 & 0.0969 & 0.0839 & 0.0757 & 0.0688 \\
\hline & $A_{n}^{2}$ & 1.0862 & 0.9124 & 0.7889 & 0.6593 & 0.5838 & 0.5291 & 0.4830 \\
\hline & $U_{n}^{2}$ & 0.1657 & 0.1378 & 0.1177 & 0.0969 & 0.0839 & 0.0757 & 0.0688 \\
\hline & $L_{n}$ & 1.1224 & 1.0239 & 0.9438 & 0.8674 & 0.8178 & 0.7806 & 0.7533 \\
\hline \multirow[t]{5}{*}{80} & $D_{n}$ & 0.1060 & 0.0990 & 0.0922 & 0.0856 & 0.0813 & 0.0778 & 0.0750 \\
\hline & $W_{n}^{2}$ & 0.1630 & 0.1393 & 0.1174 & 0.0966 & 0.0844 & 0.0756 & 0.0697 \\
\hline & $A_{n}^{2}$ & 1.0993 & 0.9148 & 0.7907 & 0.6549 & 0.5796 & 0.5264 & 0.4830 \\
\hline & $U_{n}^{2}$ & 0.1630 & 0.1393 & 0.1174 & 0.0966 & 0.0844 & 0.0756 & 0.0697 \\
\hline & $L_{n}$ & 1.0954 & 0.9992 & 0.9331 & 0.8530 & 0.8045 & 0.7685 & 0.7395 \\
\hline \multirow[t]{5}{*}{90} & $D_{n}$ & 0.1016 & 0.0943 & 0.0878 & 0.0816 & 0.0772 & 0.0737 & 0.0708 \\
\hline & $W_{n}^{2}$ & 0.1703 & 0.1411 & 0.1176 & 0.0979 & 0.0858 & 0.0768 & 0.0693 \\
\hline & $A_{n}^{2}$ & 1.1124 & 0.9441 & 0.7974 & 0.6580 & 0.5812 & 0.5287 & 0.4892 \\
\hline & $U_{n}^{2}$ & 0.1703 & 0.1411 & 0.1176 & 0.0979 & 0.0858 & 0.0768 & 0.0693 \\
\hline & $L_{\eta}$ & 1.0961 & 1.0028 & 0.9263 & 0.8426 & 0.7938 & 0.7613 & 0.7333 \\
\hline \multirow[t]{5}{*}{100} & $D_{n}$ & 0.0967 & 0.0896 & 0.0837 & 0.0774 & 0.0733 & 0.0700 & 0.0675 \\
\hline & $W_{n}^{2}$ & 0.1708 & 0.1389 & 0.1187 & 0.0971 & 0.0850 & 0.0761 & 0.0692 \\
\hline & $A_{n}^{2}$ & 1.1280 & 0.9241 & 0.7936 & 0.6629 & 0.5827 & 0.5279 & 0.4832 \\
\hline & $U_{n}^{2}$ & 0.1708 & 0.1389 & 0.1187 & 0.0971 & 0.0850 & 0.0761 & 0.0692 \\
\hline & $L_{n}$ & 1.0822 & 0.9851 & 0.9127 & 0.8377 & 0.7865 & 0.7506 & 0.7211 \\
\hline
\end{tabular}

From table 1, we can observe that critical values for all the five statistics i.e. $D_{n}, W_{n}^{2}, A_{n}^{2}, U_{n}^{2}$ and $L_{n}$ decreases as the significance level $\gamma$ increases. For Kolmogorov-Smirnov $D_{n}$ statistic, critical values decreases as sample size become larger. We can see that Waston test which is the modified form of Cramer-von Mises test behave exactly same as Cramer-von Mises test for MOR distribution. Thus we cannot distinguish between Cramer-von Mises $W_{n}^{2}$ statistic and Watson $U_{n}^{2}$ statistic for Marshal-Olkin extended Rayleigh distribution.

There is no general behavior of Anderson-Darling $A_{n}^{2}$ statistic with regard to sample size. Critical values for Liao and Shimokawa $L_{n}$ statistic also decreases as sample sizes increases for all significance levels.

\section{Power study for Marshal-Olkin extended Rayleigh distribution}

In testing of hypothesis, power is a useful tool to evaluate the goodness of a particular test or to compare two competing tests. Power of goodness of a test is denoted by $1-\beta$ and is defined as a probability that a statistic leads to reject a null hypothesis $H_{0}$, when infact it is not true. Here $\beta$ is the probability of making type-II error.

For power comparison of Marshal-Olkin Extended Rayleigh (MOR) distribution, we choose eight competitive probability distributions as the alternative to our hypothesized distribution. These distributions are:

(i). Two-parameter Weibull distribution $W(5,1)$.

(ii). Standard Cauchy distribution $C(0,1)$.

(iii). Gamma distribution $G(2,1)$.

(iv). Logistic distribution $\operatorname{LOG}(0,1)$.

(v). Exponential distribution $E(0.67)$.

(vi). Generalized Exponential distribution $G E(3,2)$.

(vii). Rayleigh distribution $R(1)$.

(viii). Generalized Rayleigh distribution $G R(0.5,1)$.

We calculated the power of Marshal-Olkin Extended Rayleigh distribution by simulating the data from the alternative distribution $\left(H_{1}\right)$ and fitting the MOR distribution $\left(H_{0}\right)$ to this data. We repeated this process 10,000 time for sample size $n=5(5), 30$ and significance level $\gamma=0.01,0.25,0.05,0.10,0.15,0.20,0.25$. We consider $n=5$ as small, $n=15$ as moderate and $n=30$ as large sample sizes. We observed the number of times each test statistics exceeded respective critical values at each level of significance to obtain power of tests. The results of power of these tests are presented in table 2 to 7 : 
Table 2: Power function when the alternative distribution is

Two parameter Weibull distribution $W(5,1)$

\begin{tabular}{|c|c|c|c|c|c|c|c|c|}
\hline \multicolumn{9}{|c|}{ Power of the Test } \\
\hline \multirow[t]{2}{*}{ Sample Size } & \multirow[t]{2}{*}{ Test Statistic } & \multicolumn{7}{|c|}{ Significance Level $\gamma$} \\
\hline & & 0.01 & 0.025 & 0.05 & 0.10 & 0.15 & 0.20 & 0.25 \\
\hline \multirow[t]{5}{*}{5} & $D_{n}$ & 0.9502 & 0.9663 & 0.9757 & 0.9843 & 0.9882 & 0.9919 & 0.9940 \\
\hline & $W_{n}^{2}$ & 0.9543 & 0.9701 & 0.9781 & 0.9868 & 0.9907 & 0.9935 & 0.9950 \\
\hline & $A_{n}^{2}$ & 0.8381 & 0.9159 & 0.9534 & 0.9741 & 0.9831 & 0.9883 & 0.9915 \\
\hline & $U_{n}^{2}$ & 0.7295 & 0.8263 & 0.8837 & 0.9319 & 0.9544 & 0.9671 & 0.9745 \\
\hline & $L_{n}$ & 0.0006 & 0.0820 & 0.4066 & 0.7531 & 0.8740 & 0.9208 & 0.9460 \\
\hline \multirow[t]{5}{*}{10} & $D_{n}$ & 0.9958 & 0.9975 & 0.9980 & 0.9990 & 0.9993 & 0.9994 & 0.9995 \\
\hline & $W_{n}^{2}$ & 0.9972 & 0.9985 & 0.9994 & 0.9996 & 0.9997 & 0.9998 & 0.9998 \\
\hline & $A_{n}^{2}$ & 0.9933 & 0.9972 & 0.9986 & 0.9993 & 0.9995 & 0.9998 & 0.9998 \\
\hline & $U_{n}^{2}$ & 0.9599 & 0.9823 & 0.9913 & 0.9966 & 0.9979 & 0.9987 & 0.9991 \\
\hline & $L_{n}$ & 0.4535 & 0.8706 & 0.9656 & 0.9920 & 0.9968 & 0.9981 & 0.9988 \\
\hline \multirow[t]{5}{*}{15} & $D_{n}$ & 0.9995 & 0.9998 & 0.9999 & 0.9999 & 0.9999 & 0.9999 & 0.9999 \\
\hline & $W_{n}^{2}$ & 0.9999 & 0.9999 & 0.9999 & 0.9999 & 0.9999 & 0.9999 & 0.9999 \\
\hline & $A_{n}^{2}$ & 0.9998 & 0.9999 & 0.9999 & 0.9999 & 0.9999 & 0.9999 & 0.9999 \\
\hline & $U_{n}^{2}$ & 0.9960 & 0.9986 & 0.9994 & 0.9998 & 0.9999 & 0.9999 & 0.9999 \\
\hline & $L_{n}$ & 0.9332 & 0.9946 & 0.9992 & 0.9999 & 0.9999 & 0.9999 & 0.9999 \\
\hline \multirow[t]{5}{*}{20} & $D_{n}$ & 0.9998 & 0.9999 & 0.9999 & 0.9999 & 0.9999 & 0.9999 & 0.9999 \\
\hline & $W_{n}^{2}$ & 0.9998 & 0.9999 & 0.9999 & 0.9999 & 0.9999 & 0.9999 & 0.9999 \\
\hline & $A_{n}^{2}$ & 0.9998 & 0.9999 & 0.9999 & 0.9999 & 0.9999 & 0.9999 & 0.9999 \\
\hline & $U_{n}^{2}$ & 0.9996 & 0.9997 & 0.9998 & 0.9999 & 0.9999 & 0.9999 & 0.9999 \\
\hline & $L_{n}$ & 0.9989 & 0.9998 & 0.9998 & 0.9999 & 0.9999 & 0.9999 & 0.9999 \\
\hline \multirow[t]{5}{*}{25} & $D_{n}$ & 0.9999 & 0.9999 & 0.9999 & 0.9999 & 0.9999 & 0.9999 & 0.9999 \\
\hline & $W_{n}^{2}$ & 0.9999 & 0.9999 & 0.9999 & 0.9999 & 0.9999 & 0.9999 & 0.9999 \\
\hline & $A_{n}^{2}$ & 0.9999 & 0.9999 & 0.9999 & 0.9999 & 0.9999 & 0.9999 & 0.9999 \\
\hline & $U_{n}^{2}$ & 0.9999 & 0.9999 & 0.9999 & 0.9999 & 0.9999 & 0.9999 & 0.9999 \\
\hline & $L_{n}$ & 0.9998 & 0.9999 & 0.9999 & 0.9999 & 0.9999 & 0.9999 & 0.9999 \\
\hline \multirow[t]{5}{*}{30} & $D_{n}$ & 0.9999 & 0.9999 & 0.9999 & 0.9999 & 0.9999 & 0.9999 & 0.9999 \\
\hline & $W_{n}^{2}$ & 0.9999 & 0.9999 & 0.9999 & 0.9999 & 0.9999 & 0.9999 & 0.9999 \\
\hline & $A_{n}^{2}$ & 0.9999 & 0.9999 & 0.9999 & 0.9999 & 0.9999 & 0.9999 & 0.9999 \\
\hline & $U_{n}^{2}$ & 0.9999 & 0.9999 & 0.9999 & 0.9999 & 0.9999 & 0.9999 & 0.9999 \\
\hline & $L_{n}$ & 0.9999 & 0.9999 & 0.9999 & 0.9999 & 0.9999 & 0.9999 & 0.9999 \\
\hline
\end{tabular}

Table 2 shows that power of all goodness of fit tests for MOR distribution increases monotonically as level of significance increases. For small to moderate sample size $W_{n}^{2}$ is more powerful than $D_{n}, A_{n}^{2}, U_{n}^{2}, L_{n}$. For large sample size all test statistics depict good power.

We computed the power of the test for Cauchy, Gamma and Logistic distribution using $D_{n}, W_{n}^{2}, A_{n}^{2}, U_{n}^{2}$ and $L_{n}$ statistics. The results of power of the tests are very close to one even for small sample sizes for different choices of probability of type-I error. Thus, those alternative distributions are not much informative 
Table 3: Power function when the alternative distribution is Exponential distribution

\begin{tabular}{|c|c|c|c|c|c|c|c|c|}
\hline \multicolumn{9}{|c|}{ Power of the Test } \\
\hline \multirow[t]{2}{*}{ Sample Size } & \multirow{2}{*}{$\begin{array}{l}\text { Test } \\
\text { Statistic }\end{array}$} & \multicolumn{7}{|c|}{ Significance Level $\gamma$} \\
\hline & & 0.01 & 0.025 & 0.05 & 0.10 & 0.15 & 0.20 & 0.25 \\
\hline \multirow[t]{5}{*}{5} & $D_{n}$ & 0.8206 & 0.8551 & 0.8823 & 0.9131 & 0.9291 & 0.9403 & 0.9479 \\
\hline & $W_{n}^{2}$ & 0.7817 & 0.8386 & 0.8767 & 0.9114 & 0.9331 & 0.9441 & 0.9528 \\
\hline & $A_{n}^{2}$ & 0.9005 & 0.9278 & 0.9441 & 0.9620 & 0.9700 & 0.9761 & 0.9799 \\
\hline & $U_{n}^{2}$ & 0.4668 & 0.5476 & 0.6282 & 0.7259 & 0.7819 & 0.8185 & 0.8447 \\
\hline & $L_{n}$ & 0.8312 & 0.8860 & 0.9252 & 0.9493 & 0.9623 & 0.9702 & 0.9763 \\
\hline \multirow[t]{5}{*}{10} & $D_{n}$ & 0.8893 & 0.9172 & 0.9364 & 0.9526 & 0.9627 & 0.9697 & 0.9807 \\
\hline & $W_{n}^{2}$ & 0.8956 & 0.9244 & 0.9453 & 0.9648 & 0.9740 & 0.9807 & 0.9855 \\
\hline & $A_{n}^{2}$ & 0.9733 & 0.9835 & 0.9892 & 0.9934 & 0.9951 & 0.9959 & 0.9966 \\
\hline & $U_{n}^{2}$ & 0.6848 & 0.7703 & 0.8276 & 0.8824 & 0.9132 & 0.9284 & 0.9440 \\
\hline & $L_{n}$ & 0.9716 & 0.9851 & 0.9905 & 0.9936 & 0.9958 & 0.9972 & 0.9975 \\
\hline \multirow[t]{5}{*}{15} & $D_{n}$ & 0.9381 & 0.9602 & 0.9703 & 0.9852 & 0.9896 & 0.9919 & 0.9935 \\
\hline & $W_{n}^{2}$ & 0.9520 & 0.9712 & 0.9810 & 0.9885 & 0.9925 & 0.9941 & 0.9956 \\
\hline & $A_{n}^{2}$ & 0.9945 & 0.9960 & 0.9974 & 0.9985 & 0.9988 & 0.9992 & 0.9995 \\
\hline & $U_{n}^{2}$ & 0.8278 & 0.8892 & 0.9226 & 0.9513 & 0.9664 & 0.9746 & 0.9797 \\
\hline & $L_{n}$ & 0.9950 & 0.9977 & 0.9983 & 0.9993 & 0.9996 & 0.9997 & 0.9998 \\
\hline \multirow[t]{5}{*}{20} & $D_{n}$ & 0.9714 & 0.9842 & 0.9902 & 0.9946 & 0.9966 & 0.9981 & 0.9986 \\
\hline & $W_{n}^{2}$ & 0.9804 & 0.9892 & 0.9939 & 0.9973 & 0.9985 & 0.9991 & 0.9994 \\
\hline & $A_{n}^{2}$ & 0.9989 & 0.9997 & 0.9998 & 0.9998 & 0.9998 & 0.9998 & 0.9998 \\
\hline & $U_{n}^{2}$ & 0.9165 & 0.9506 & 0.9689 & 0.9833 & 0.9891 & 0.9932 & 0.9952 \\
\hline & $L_{n}$ & 0.9994 & 0.9997 & 0.9998 & 0.9998 & 0.9998 & 0.9998 & 0.9999 \\
\hline \multirow[t]{5}{*}{25} & $D_{n}$ & 0.9904 & 0.9941 & 0.9964 & 0.9985 & 0.9988 & 0.9991 & 0.9992 \\
\hline & $W_{n}^{2}$ & 0.9934 & 0.9971 & 0.9981 & 0.9994 & 0.9995 & 0.9997 & 0.9997 \\
\hline & $A_{n}^{2}$ & 0.9995 & 0.9996 & 0.9997 & 0.9998 & 0.9998 & 0.9998 & 0.9998 \\
\hline & $U_{n}^{2}$ & 0.9642 & 0.9827 & 0.9903 & 0.9951 & 0.9969 & 0.9979 & 0.9986 \\
\hline & $L_{n}$ & 0.9997 & 0.9997 & 0.9997 & 0.9998 & 0.9998 & 0.9999 & 0.9999 \\
\hline \multirow[t]{5}{*}{30} & $D_{n}$ & 0.9960 & 0.9975 & 0.9987 & 0.9992 & 0.9997 & 0.9997 & 0.9999 \\
\hline & $W_{n}^{2}$ & 0.9976 & 0.9985 & 0.9990 & 0.9997 & 0.9998 & 0.9999 & 0.9999 \\
\hline & $A_{n}^{2}$ & 0.9997 & 0.9998 & 0.9998 & 0.9999 & 0.9999 & 0.9999 & 0.9999 \\
\hline & $U_{n}^{2}$ & 0.9849 & 0.9924 & 0.9954 & 0.9978 & 0.9985 & 0.9990 & 0.9994 \\
\hline & $L_{n}$ & 0.9998 & 0.9999 & 0.9999 & 0.9999 & 0.9999 & 0.9999 & 0.9999 \\
\hline
\end{tabular}

From table 3 we have observed that with increase in significance level, power of all goodness of fit tests for MOR distribution increases. For all sample sizes, $L_{n}$ is more powerful than $D_{n}, W_{n}^{2}, A_{n}^{2} U_{n}^{2}$. All test statistics present high power for moderate and large sample sizes. $U_{n}^{2}$ represented comparatively low power than other tests for all sample sizes.

Table 4: Power function when the alternative distribution is Generalized Exponential distribution

\begin{tabular}{|c|c|c|c|c|c|c|c|c|}
\hline \multicolumn{9}{|c|}{ Power of the Test } \\
\hline \multirow[t]{2}{*}{ Sample Size } & \multirow[t]{2}{*}{$\begin{array}{l}\text { Test } \\
\text { Statistic }\end{array}$} & \multicolumn{7}{|c|}{$\begin{array}{c}\text { Significance }+ \\
\text { Level } \gamma\end{array}$} \\
\hline & & 0.01 & 0.025 & 0.05 & 0.10 & 0.15 & 0.20 & 0.25 \\
\hline \multirow[t]{5}{*}{5} & $D_{n}$ & 0.5656 & 0.6390 & 0.6985 & 0.7645 & 0.8055 & 0.8330 & 0.8557 \\
\hline & $W_{n}^{2}$ & 0.5352 & 0.6186 & 0.6931 & 0.7608 & 0.8050 & 0.8347 & 0.8585 \\
\hline & $A_{n}^{2}$ & 0.4871 & 0.5856 & 0.6684 & 0.7546 & 0.8017 & 0.8355 & 0.8594 \\
\hline & $U_{n}^{2}$ & 0.1390 & 0.2163 & 0.3057 & 0.4887 & 0.4887 & 0.5528 & 0.6034 \\
\hline & $L_{n}$ & 0.2352 & 0.3608 & 0.4826 & 0.5992 & 0.6777 & 0.7340 & 0.7734 \\
\hline \multirow[t]{3}{*}{10} & $D_{n}$ & 0.5868 & 0.6526 & 0.7106 & 0.7706 & 0.8127 & 0.8420 & 0.8653 \\
\hline & $W_{n}^{2}$ & 0.6085 & 0.6700 & 0.7263 & 0.7899 & 0.8293 & 0.8598 & 0.8793 \\
\hline & $A_{n}^{2}$ & 0.5938 & 0.6755 & 0.7392 & 0.8063 & 0.8483 & 0.8771 & 0.8949 \\
\hline
\end{tabular}




\begin{tabular}{|c|c|c|c|c|c|c|c|c|}
\hline \multirow{5}{*}{15} & $U_{n}^{2}$ & 0.1422 & 0.2179 & 0.3035 & 0.4182 & 0.5024 & 0.5661 & 0.6226 \\
\cline { 2 - 8 } & $L_{n}$ & 0.3997 & 0.5459 & 0.6424 & 0.7423 & 0.7936 & 0.8318 & 0.8613 \\
\cline { 2 - 8 } & $D_{n}$ & 0.6075 & 0.6735 & 0.7355 & 0.8013 & 0.8386 & 0.8668 & 0.8883 \\
\cline { 2 - 8 } & $W_{n}^{2}$ & 0.6558 & 0.7157 & 0.7671 & 0.8282 & 0.8590 & 0.8825 & 0.9022 \\
\cline { 2 - 8 } & $A_{n}^{2}$ & 0.6824 & 0.7464 & 0.7951 & 0.8534 & 0.8840 & 0.9064 & 0.9224 \\
\cline { 2 - 8 } & $U_{n}^{2}$ & 0.1554 & 0.2324 & 0.3071 & 0.4267 & 0.5096 & 0.5781 & 0.6311 \\
\hline \multirow{6}{*}{20} & $L_{n}$ & 0.5735 & 0.6755 & 0.7520 & 0.8237 & 0.8604 & 0.8851 & 0.9040 \\
\cline { 2 - 8 } & $D_{n}$ & 0.6589 & 0.7303 & 0.7816 & 0.8338 & 0.8669 & 0.8892 & 0.9082 \\
\cline { 2 - 8 } & $W_{n}^{2}$ & 0.7150 & 0.7649 & 0.8116 & 0.8581 & 0.8859 & 0.9063 & 0.9220 \\
\cline { 2 - 8 } & $A_{n}^{2}$ & 0.7379 & 0.8003 & 0.8424 & 0.8892 & 0.9131 & 0.9292 & 0.9424 \\
\cline { 2 - 8 } & $U_{n}^{2}$ & 0.1659 & 0.2407 & 0.3390 & 0.4574 & 0.5424 & 0.6065 & 0.6607 \\
\cline { 2 - 8 } & $L_{n}$ & 0.6843 & 0.7754 & 0.8291 & 0.8749 & 0.9024 & 0.9180 & 0.9322 \\
\cline { 2 - 8 } & $D_{n}$ & 0.7206 & 0.7800 & 0.8254 & 0.8702 & 0.8974 & 0.9154 & 0.9292 \\
\cline { 2 - 8 } & $W_{n}^{2}$ & 0.7632 & 0.8160 & 0.8543 & 0.8936 & 0.9140 & 0.9308 & 0.9432 \\
\cline { 2 - 8 } & $A_{n}^{2}$ & 0.7931 & 0.8545 & 0.8879 & 0.9193 & 0.9386 & 0.9516 & 0.9615 \\
\cline { 2 - 8 } & $U_{n}^{2}$ & 0.1835 & 0.2810 & 0.3753 & 0.4926 & 0.5717 & 0.6299 & 0.6845 \\
\hline \multirow{6}{*}{30} & $L_{n}$ & 0.7788 & 0.8389 & 0.8789 & 0.9142 & 0.9334 & 0.9472 & 0.9564 \\
\cline { 2 - 8 } & $D_{n}$ & 0.7527 & 0.8047 & 0.8465 & 0.8879 & 0.9147 & 0.9296 & 0.9427 \\
\cline { 2 - 8 } & $W_{n}^{2}$ & 0.8049 & 0.8471 & 0.8750 & 0.9090 & 0.9298 & 0.9438 & 0.9545 \\
\cline { 2 - 8 } & $A_{n}^{2}$ & 0.8426 & 0.8831 & 0.9105 & 0.9362 & 0.9517 & 0.9628 & 0.9696 \\
\cline { 2 - 8 } & $U_{n}^{2}$ & 0.2168 & 0.3040 & 0.3855 & 0.5041 & 0.5839 & 0.6457 & 0.6973 \\
\cline { 2 - 8 } & $L_{n}$ & 0.8281 & 0.8759 & 0.9091 & 0.9354 & 0.9488 & 0.9599 & 0.9673 \\
\hline
\end{tabular}

Table 4 presented that for MOR distribution, power of tests improves as significance level increases. We observed that for small sample sizes $D_{n}$ and $W_{n}^{2}$ show high power as compared to other tests while Anderson-Darling statistic $A_{n}^{2}$ appears to be more powerful than $D_{n}, W_{n}^{2}, U_{n}^{2}, L_{n}$ for moderate and large sample sizes.

Table 5: Power function when the alternative distribution is Rayleigh distribution

\begin{tabular}{|c|c|c|c|c|c|c|c|c|}
\hline \multicolumn{9}{|c|}{ Power of the Test } \\
\hline \multirow[t]{2}{*}{ Sample Size } & \multirow[t]{2}{*}{ Test Statistic } & \multicolumn{7}{|c|}{$\begin{array}{l}\text { Significance Level } \gamma \\
\end{array}$} \\
\hline & & 0.01 & 0.025 & 0.05 & 0.10 & 0.15 & 0.20 & 0.25 \\
\hline \multirow[t]{5}{*}{5} & $D_{n}$ & 0.8863 & 0.9098 & 0.9270 & 0.9472 & 0.9565 & 0.9634 & 0.9692 \\
\hline & $W_{n}^{2}$ & 0.8807 & 0.9085 & 0.9258 & 0.9453 & 0.9567 & 0.9654 & 0.9709 \\
\hline & $A_{n}^{2}$ & 0.8692 & 0.9078 & 0.9298 & 0.9510 & 0.9629 & 0.9696 & 0.9747 \\
\hline & $U_{n}^{2}$ & 0.4153 & 0.5151 & 0.5902 & 0.6896 & 0.7476 & 0.7907 & 0.8222 \\
\hline & $L_{n}$ & 0.6107 & 0.7729 & 0.8593 & 0.9185 & 0.9417 & 0.9547 & 0.9632 \\
\hline \multirow[t]{5}{*}{10} & $D_{n}$ & 0.9662 & 0.9740 & 0.9812 & 0.9870 & 0.9901 & 0.9923 & 0.9945 \\
\hline & $W_{n}^{2}$ & 0.9723 & 0.9798 & 0.9846 & 0.9896 & 0.9918 & 0.9941 & 0.9956 \\
\hline & $A_{n}^{2}$ & 0.9740 & 0.9840 & 0.9890 & 0.9932 & 0.9947 & 0.9960 & 0.9966 \\
\hline & $U_{n}^{2}$ & 0.6222 & 0.7184 & 0.7910 & 0.8557 & 0.8957 & 0.9189 & 0.9364 \\
\hline & $L_{n}$ & 0.9232 & 0.9665 & 0.9814 & 0.9906 & 0.9936 & 0.9946 & 0.9956 \\
\hline \multirow[t]{5}{*}{15} & $D_{n}$ & 0.9893 & 0.9925 & 0.9958 & 0.9968 & 0.9980 & 0.9982 & 0.9984 \\
\hline & $W_{n}^{2}$ & 0.9928 & 0.9956 & 0.9969 & 0.9981 & 0.9984 & 0.9987 & 0.9987 \\
\hline & $A_{n}^{2}$ & 0.9939 & 0.9968 & 0.9978 & 0.9988 & 0.9989 & 0.9990 & 0.9991 \\
\hline & $U_{n}^{2}$ & 0.7689 & 0.8475 & 0.8942 & 0.9363 & 0.9542 & 0.9666 & 0.9749 \\
\hline & $L_{n}$ & 0.9848 & 0.9940 & 0.9970 & 0.9985 & 0.9990 & 0.9991 & 0.9994 \\
\hline \multirow[t]{5}{*}{20} & $D_{n}$ & 0.9975 & 0.9984 & 0.9986 & 0.9992 & 0.9995 & 0.9996 & 0.9998 \\
\hline & $W_{n}^{2}$ & 0.9985 & 0.9989 & 0.9992 & 0.9995 & 0.9996 & 0.9996 & 0.9996 \\
\hline & $A_{n}^{2}$ & 0.9988 & 0.9992 & 0.9992 & 0.9995 & 0.9996 & 0.9997 & 0.9998 \\
\hline & $U_{n}^{2}$ & 0.8689 & 0.9195 & 0.9505 & 0.9753 & 0.9838 & 0.9894 & 0.9928 \\
\hline & $L_{n}$ & 0.9982 & 0.9991 & 0.9993 & 0.9996 & 0.9997 & 0.9997 & 0.9998 \\
\hline 25 & $D_{n}$ & 0.9995 & 0.9996 & 0.9997 & 0.9998 & 0.9998 & 0.9999 & 0.9999 \\
\hline
\end{tabular}




\begin{tabular}{|c|c|c|c|c|c|c|c|c|}
\hline \multirow{5}{*}{} & $W_{n}^{2}$ & 0.9997 & 0.9998 & 0.9998 & 0.9998 & 0.9998 & 0.9999 & 0.9999 \\
\cline { 2 - 8 } & $A_{n}^{2}$ & 0.9998 & 0.9999 & 0.9999 & 0.9999 & 0.9999 & 0.9999 & 0.9999 \\
\cline { 2 - 9 } & $U_{n}^{2}$ & 0.9281 & 0.9622 & 0.9783 & 0.9893 & 0.9936 & 0.9962 & 0.9975 \\
\cline { 2 - 8 } & $L_{n}$ & 0.9998 & 0.9999 & 0.9999 & 0.9999 & 0.9999 & 0.9999 & 0.9999 \\
\hline \multirow{3}{*}{30} & $D_{n}$ & 0.9998 & 0.9998 & 0.9998 & 0.9999 & 0.9999 & 0.9999 & 0.9999 \\
\cline { 2 - 8 } & $W_{n}^{2}$ & 0.9998 & 0.9998 & 0.9999 & 0.9999 & 0.9999 & 0.9999 & 0.9999 \\
\cline { 2 - 8 } & $A_{n}^{2}$ & 0.9997 & 0.9999 & 0.9999 & 0.9999 & 0.9999 & 0.9999 & 0.9999 \\
\cline { 2 - 8 } & $U_{n}^{2}$ & 0.9655 & 0.9827 & 0.9901 & 0.9962 & 0.9981 & 0.9990 & 0.9993 \\
\cline { 2 - 8 } & $L_{n}$ & 0.9997 & 0.9998 & 0.9999 & 0.9999 & 0.9999 & 0.9999 & 0.9999 \\
\hline
\end{tabular}

Table 5 showed that for sample size as $\mathrm{n}=5$ and significance level $\gamma=0.01$ and $\gamma=0.025$, Kolmogorov-Smirnov $D_{n}$ statistic appears to be more powerful than other tests but for the same sample size and significance level 0.05 to 0.25 , Anderson-Darling $A_{n}^{2}$ depicts high power than $D_{n}, W_{n}^{2}, U_{n}^{2}, L_{n}$. For all the significance levels and sample sizes from 10 till 30, $A_{n}^{2}$ is the most powerful among all test statistics except for $\mathrm{n}=30, \gamma=0.01$.

Table 6: Power function when the alternative distribution is

Generalized Rayleigh distribution

\begin{tabular}{|c|c|c|c|c|c|c|c|c|}
\hline \multicolumn{9}{|c|}{ Power of the Test } \\
\hline \multirow[t]{2}{*}{ Sample Size } & \multirow[t]{2}{*}{ Test Statistic } & \multicolumn{7}{|c|}{ Significance Level $\gamma$} \\
\hline & & 0.01 & 0.025 & 0.05 & 0.10 & 0.15 & 0.20 & 0.25 \\
\hline \multirow[t]{5}{*}{5} & $D_{n}$ & 0.5696 & 0.6359 & 0.6900 & 0.7651 & 0.8087 & 0.8360 & 0.8586 \\
\hline & $W_{n}^{2}$ & 0.5384 & 0.6141 & 0.6762 & 0.7539 & 0.8030 & 0.8375 & 0.8622 \\
\hline & $A_{n}^{2}$ & 0.5131 & 0.6176 & 0.6960 & 0.7743 & 0.8206 & 0.8536 & 0.8768 \\
\hline & $U_{n}^{2}$ & 0.1688 & 0.2421 & 0.3169 & 0.4311 & 0.5059 & 0.5654 & 0.6197 \\
\hline & $L_{n}$ & 0.2638 & 0.4240 & 0.5530 & 0.6788 & 0.7524 & 0.7912 & 0.8237 \\
\hline \multirow[t]{5}{*}{10} & $D_{n}$ & 0.5657 & 0.6383 & 0.6925 & 0.7613 & 0.7995 & 0.8350 & 0.8631 \\
\hline & $W_{n}^{2}$ & 0.5779 & 0.6507 & 0.7162 & 0.7854 & 0.8279 & 0.8589 & 0.8789 \\
\hline & $A_{n}^{2}$ & 0.6299 & 0.7062 & 0.7697 & 0.8351 & 0.8699 & 0.8941 & 0.9129 \\
\hline & $U_{n}^{2}$ & 0.1955 & 0.2820 & 0.3726 & 0.4822 & 0.5612 & 0.6209 & 0.6645 \\
\hline & $L_{n}$ & 0.4916 & 0.6390 & 0.7319 & 0.8144 & 0.8575 & 0.8813 & 0.9011 \\
\hline \multirow[t]{5}{*}{15} & $D_{n}$ & 0.5717 & 0.6510 & 0.7216 & 0.7850 & 0.8270 & 0.8573 & 0.8846 \\
\hline & $W_{n}^{2}$ & 0.6076 & 0.6823 & 0.7457 & 0.8113 & 0.8496 & 0.8790 & 0.8992 \\
\hline & $A_{n}^{2}$ & 0.6900 & 0.7683 & 0.8160 & 0.8731 & 0.9004 & 0.9219 & 0.9371 \\
\hline & $U_{n}^{2}$ & 0.2151 & 0.3121 & 0.4064 & 0.5154 & 0.5931 & 0.6498 & 0.6949 \\
\hline & $L_{n}$ & 0.6160 & 0.7461 & 0.8183 & 0.8785 & 0.9067 & 0.9232 & 0.9381 \\
\hline \multirow[t]{5}{*}{20} & $D_{n}$ & 0.6223 & 0.6905 & 0.7526 & 0.8131 & 0.8511 & 0.8804 & 0.8999 \\
\hline & $W_{n}^{2}$ & 0.6517 & 0.7219 & 0.7812 & 0.8418 & 0.8773 & 0.8988 & 0.9164 \\
\hline & $A_{n}^{2}$ & 0.7399 & 0.8145 & 0.8577 & 0.8964 & 0.9195 & 0.9382 & 0.9489 \\
\hline & $U_{n}^{2}$ & 0.2509 & 0.3468 & 0.4441 & 0.5584 & 0.6301 & 0.6838 & 0.7290 \\
\hline & $L_{n}$ & 0.7381 & 0.8175 & 0.8692 & 0.9067 & 0.9298 & 0.9437 & 0.9544 \\
\hline \multirow[t]{5}{*}{25} & $D_{n}$ & 0.6594 & 0.7216 & 0.7803 & 0.8394 & 0.8745 & 0.8991 & 0.9157 \\
\hline & $W_{n}^{2}$ & 0.6941 & 0.7615 & 0.8192 & 0.8696 & 0.8975 & 0.9203 & 0.9342 \\
\hline & $A_{n}^{2}$ & 0.7782 & 0.8377 & 0.8791 & 0.9174 & 0.9390 & 0.9517 & 0.9618 \\
\hline & $U_{n}^{2}$ & 0.2934 & 0.3945 & 0.4917 & 0.5996 & 0.6666 & 0.7250 & 0.7661 \\
\hline & $L_{n}$ & 0.7777 & 0.8488 & 0.8919 & 0.9281 & 0.9470 & 0.9573 & 0.9657 \\
\hline \multirow[t]{5}{*}{30} & $D_{n}$ & 0.6898 & 0.7547 & 0.8083 & 0.8645 & 0.8965 & 0.9170 & 0.9321 \\
\hline & $W_{n}^{2}$ & 0.7245 & 0.7932 & 0.8373 & 0.8876 & 0.9151 & 0.9343 & 0.9471 \\
\hline & $A_{n}^{2}$ & 0.8253 & 0.8688 & 0.9060 & 0.9386 & 0.9532 & 0.9652 & 0.9736 \\
\hline & $U_{n}^{2}$ & 0.3169 & 0.4358 & 0.5306 & 0.6452 & 0.7138 & 0.7624 & 0.7988 \\
\hline & $L_{n}$ & 0.8296 & 0.8883 & 0.9218 & 0.9484 & 0.9626 & 0.9710 & 0.9773 \\
\hline
\end{tabular}

From Table 6 we can see that for $\mathrm{n}=5$ and $\gamma=0.01,0.025$, Kolmogorov-Smirnov $D_{n}$ statistic is more powerful than other tests but for the same sample size with $\gamma=0.05,0.10,0.15,0.20,0.25$, Anderson-Darling $A_{n}^{2}$ shows high power 
than $D_{n}, W_{n}^{2}, U_{n}^{2}, L_{n}$.While for all the significance levels and sample size $\mathrm{n}=10, A_{n}^{2}$ is the most powerful among all test statistics. We also observe that for sample size $15, A_{n}^{2}$ represents highest power for $\gamma=0.01,0.025$ while for $\gamma=$ 0.05 till $\gamma=0.25, L_{n}$ is more powerful. For sample size $20,25 L_{n}$ appears to be most powerful except $\gamma=0.01$ and for $\mathrm{n}=30$ and $\gamma=0.01, L_{n}$ is most powerful among all statistics.

\section{Real life application}

In this section, we used a real life application using well known data set to show the wider applicability of our proposed model over other competing models in survival and reliability. We considered Exponential, Rayleigh, Generalized Exponential (GE) (Gupta and Kundu, 2001), Generalized Rayleigh(GR)(Kundu and Raqab, 2005) distributions.

The data set is about the strengths of $1.5 \mathrm{~cm}$ glass fibers, measured at the National Physical Laboratory, England. The data is taken from Smith and Naylor (1987) comprises of 63 observations: $0.55,0.93,1.25,1.36,1.49,1.52$, $1.58,1.61,1.64,1.68,1.73,1.81,2,0.74,1.04,1.27,1.39,1.49,1.53,1.59,1.61,1.66,1.68,1.76,1.82,2.01,0.77$, $1.11,1.28,1.42,1.5,1.54,1.6,1.62,1.66,1.69,1.76,1.84,2.24,0.81,1.13,1.29,1.48,1.5,1.55,1.61,1.62,1.66$, $1.7,1.77,1.84,0.84,1.24,1.3,1.48,1.51,1.55,1.61,1.63,1.67,1.7,1.78,1.89$.

For each distribution, using maximum likelihood estimates we calculated AIC (Akaike Information Criterion), BIC (Bayesian Information Criterion), KS (Kolmogorov-Smirnov) statistic with its respective p-value. Recently, the data was reanalyzed in Barreto-Souza et al. (2010) to fit the beta-generalized exponential (BGE) distribution.

Table 7. Maximum likelihood estimates along with their standard errors, log-likelihood, AIC, BIC, KolmogorovSmirnov statistics for the strengths of fibers

\begin{tabular}{|l|l|l|l|l|}
\hline Distribution & $\begin{array}{l}\text { ML Estimates } \\
\text { (Standard Errors })\end{array}$ & Log likelihood & AIC & BIC \\
\hline Weibull & $\begin{array}{l}5.78,1.62 \\
(0.03,(0.57)\end{array}$ & -15.20 & 34.41 & 38.69 \\
\hline Cauchy & $\begin{array}{l}1.59,0.02 \\
(0.13),(0.02)\end{array}$ & -17.63 & 39.27 & 49.54 \\
\hline Gamma $(\alpha, \beta)$ & $\begin{array}{l}17.4458,11.5745 \\
(3.04686),(2.072)\end{array}$ & -23.95 & 51.90 & 56.19 \\
\hline Logistic & $\begin{array}{l}1.54,0.17 \\
(0.04),(0.02)\end{array}$ & -16.02 & 36.04 & 40.33 \\
\hline Exponential $(\lambda)$ & $\begin{array}{l}0.6636 \\
(0.08361)\end{array}$ & -88.83 & 179.7 & 181.8 \\
\hline Rayleigh $(\beta)$ & $\begin{array}{l}0.6490 \\
(0.04089)\end{array}$ & -49.79 & 101.6 & 103.7 \\
\hline GE $(\alpha, \lambda)$ & $\begin{array}{l}31.349,2.612 \\
(9.519),(0.2380)\end{array}$ & -31.38 & 66.8 & 71.1 \\
\hline GR $(\alpha, \beta)$ & $\begin{array}{l}5.4860,0.9869 \\
(1.2196),(0.0549)\end{array}$ & -23.93 & 51.9 & 56.1 \\
\hline MR $(\alpha, \beta)$ & $\begin{array}{l}120.1841,1.4115 \\
(4.9197),(0.0291)\end{array}$ & -13.00 & 30 & 34.3 \\
\hline
\end{tabular}

Table 8. Kolmogorov-Smirnov Cramer Von Misses and Anderson-Darling statistics for and p-values for the strengths of fibers.

\begin{tabular}{|l|l|l|l|l|l|l|}
\hline Distribution & Dn & p- value & $W_{n}^{2}$ & p- value & $A_{n}^{2}$ & p- value \\
\hline Weibull & 0.1522 & 0.1079 & 0.2150 & 0.2404 & 1.2410 & 0.2525 \\
\hline Cauchy & 0.1029 & 0.5167 & 0.1905 & 0.2871 & 1.4001 & 0.2021 \\
\hline Gamma $(\alpha, \beta)$ & 0.2163 & 0.005508 & 0.56536 & 0.0269 & 3.0856 & 0.02498 \\
\hline Logistic & 0.1253 & 0.276 & 0.1724 & 0.3284 & 1.2846 & 0.2373 \\
\hline Exponential $(\lambda)$ & 0.418 & $<0.0001$ & 3.8622 & $<0.0001$ & 18.425 & $<0.00001$ \\
\hline Rayleigh $(\beta)$ & 0.3339 & $<0.0001$ & 2.32209 & $<0.0001$ & 11.4249 & $<0.0001$ \\
\hline
\end{tabular}




\begin{tabular}{|l|l|l|l|l|l|l|}
\hline $\operatorname{GE}(\alpha, \lambda)$ & 0.2293 & 0.0027 & 0.79862 & 0.0072 & 4.3375 & 0.0060 \\
\hline $\operatorname{GR}(\alpha, \beta)$ & 0.2151 & 0.0059 & 0.5833 & 0.02428 & 3.1297 & 0.02367 \\
\hline $\operatorname{MR}(\alpha, \beta)$ & 0.1065 & 0.4728 & 0.1068 & 0.5539 & 0.7367 & 0.5283 \\
\hline
\end{tabular}

The results of MLEs (along with their standard errors), log-likelihood, AIC and BIC are presented in table 7.

Kolmogorov-Smirnov, Cramer Von Misses and Anderson-Darling along withp-values are presented in table 8. It is evident from table 7 and 8 that Marshal-Olkin Extended Rayleigh distribution provides a better fit than the other competing models.

\section{Conclusion}

We obtained critical values for Marshal-Olkin Extended Rayleigh distribution using Monte Carlo simulations for different sample sizes $\mathrm{n}$ and significance level $\gamma$. It is visible from table of critical values that Marshal-Olkin extended Rayleigh distribution cannot distinguish between Cramer-von Mises $W_{n}^{2}$ test and Watson $U_{n}^{2}$ test. As critical values of $W_{n}^{2}$ and $U_{n}^{2}$ are same for all sample sizes at each significance level. It can clearly be observed from tables of power study that Marshal-Olkin extended Rayleigh distribution shows high power than Weibull, Cauchy, Gamma, Logistic, Exponential, Generalized Exponential, Rayleigh and Generalized Rayleigh distribution. From the real life application, it can be seen that Marshal-Olkin Extended Rayleigh distribution outperforms other existing distributions.

\section{References}

1. Abd-Elfattah, A. (2011). Goodness of fit test for the generalized rayleigh distribution with unknown parameters. Journal of Statistical Computation and Simulation, 81(3):357-366.

2. Abd-Elfattah, A., Hala, A. F., and Omima, A. (2010). Goodness of fit tests for generalized frechet distribution. Australian Journal of Basic and Applied Sciences, 4(2):286-301.

3. Al-Zahrani, B. (2012). Goodness-of-fit for the topp-leone distribution with unknown parameters. Applied Mathematical Sciences, 6(128):6355-6363.

4. Choulakian, V. and Stephens, M. (2001). Goodness-of-fit tests for the generalized pareto distribution. Technometrics, 43(4):478-484.

5. Cordeiro, G. M. and Lemonte, A. J. (2013). On the marshall-olkin extended Weibull distribution. Statistical Papers, 54(2):333-353.

6. Gupta, R. D. and Kundu, D. (1999). Generalized exponential distributions. Australian \& New Zealand Journal of Statistics, 41(2):173-188.

7. Hassan, A. S. (2005). Goodness-of-fit for the generalized exponential distribution. Interstat Electronic Journal, pages $1-15$.

8. Lilliefors, H. W. (1967). On the kolmogorov-smirnov test for normality with mean and variance unknown. Journal of the American Statistical Association, 62(318):399-402.

9. Lilliefors, H. W. (1969). On the kolmogorov-smirnov test for the exponential distribution

10. with mean unknown. Journal of the American Statistical Association, 64(325):387-389.

11. Marshall, A. W. and Olkin, I. (1997). A new method for adding a parameter to a family of distributions with application to the exponential and weibull families. Biometrika, 84(3):641-652.

12. Shin, H., Jung, Y., Jeong, C., and Heo, J.-H. (2011). Assessment of modified anderson-darling test statistics for the generalized extreme value and generalized logistic distributions.Stochastic Environmental Research and Risk Assessment, 26(1):105-114.

13. Soetaert, K. (2009). Package rootsolve: roots, gradients and steady-states in r. Woodruff, B. W., Viviano, P. J., Moore, A. H., and Dunne, E. J. (1984). Modified goodness-of-fit tests for gamma distributions with unknown location and scale parameters. Reliability, IEEE Transactions on, 33(3):241-245.

14. Yen, V. C. and Moore, A. H. (1988). Modified goodness-of-fit test for the laplace distribution. Communications in Statistics-Simulation and Computation, 17(1):275-281.

15. Zainal Abidin, Nahdiya, A. M. B. and Midi, H. (2012). The goodness-of-fit test for gumbel distribution:a comparative study. Matematika, 28(1):3548. 